\title{
Development of RECLS score to predict survival in lung cancer patients with malignant pleural effusion
}

\author{
Tianli Zhang ${ }^{1 \#}$, Xi Chen ${ }^{2 \#}$, Bing Wan ${ }^{3 \#}$, Yangyang Xu ${ }^{4}$, Hongbing Liu ${ }^{1,2,4}$, Tangfeng Lv $^{1,2,4}$, Ping Zhan ${ }^{1,2,4}$, \\ Yong Song ${ }^{1,2,4}$
}

${ }^{1}$ Department of Respiratory and Critical Care Medicine, Jinling Hospital, Medical School of Southeast University, Nanjing, China; ${ }^{2}$ Department of Respiratory and Critical Care Medicine, Jinling Hospital, Nanjing University School of Medicine, Nanjing, China; ${ }^{3}$ Department of Respiratory and Critical Care Medicine, The Affiliated Jiangning Hospital of Nanjing Medical University, Nanjing, China; ${ }^{4}$ Department of Respiratory and Critical Care Medicine, Jinling Hospital, Jinling Clinical College of Nanjing Medical University, Nanjing, China

Contributions: (I) Concept and design: T Zhang, X Chen, B Wan, T Lv, P Zhan, Y Song; (II) Administrative support: B Wan, Y Xu, H Liu; (III) Provision of study materials or patients: T Zhang, X Chen, B Wan; (IV) Collection and assembly of data: T Zhang, X Chen, B Wan, Y Xu; (V) Data analysis and interpretation: T Zhang, X Chen, B Wan, T Lv, P Zhan; (VI) Manuscript writing: All authors; (VII) Final approval of manuscript: All authors.

\#These authors contributed equally to this work.

Correspondence to: Tangfeng Lv, MD. Ping Zhan, MD. Yong Song, MD. East Zhongshan Road 305, Nanjing 210002, China.

Email: bairoushui@163.com; zhanping207@163.com; yongsong6310@yahoo.com.

Background: Malignant pleural effusion (MPE) is usually caused by lung cancer, and the prognostic factors are poorly understood. We aimed to develop models to predict the survival of lung cancer patients and lung adenocarcinoma patients with MPE.

Methods: We enrolled lung cancer patients with MPE in Nanjing Jinling Hospital from January 2008 to June 2018 into our study. We selected risk factors using multivariable Cox proportional-hazards analysis in the development cohort. The risk models were created according to the risk ratio (RR) value. The participants were categorized into low-risk, moderate-risk, and high-risk groups according to the sum of every risk factor.

Results: A total of 367 lung cancer patients were included in the development cohort. The scoring systems RECLS (relapse or not, ECOG PS, CRP, pleural LDH, and TNM stage) and RECLSAM (relapse or not, ECOG PS, CRP, pleural LDH, TNM stage, albumin-globulin ratio, and activating gene mutation) were created for lung cancer patients with MPE and lung adenocarcinoma patients with MPE. The area under the curve (AUC) values for the RECLS model were 0.911, 0.845, and 0.754, respectively, at 1 month, 6 months, and 12 months.

Conclusions: This study developed prognostic models for lung cancer patients with MPE. The RECLS and RECLSAM scores are practical, clinically applicable models to help guide the selection of optimal treatment strategies.

Keywords: Lung cancer; malignant pleural effusion; prognostic model

Submitted Nov 12, 2020. Accepted for publication Jan 29, 2021.

doi: $10.21037 /$ tlcr-20-1191

View this article at: http://dx.doi.org/10.21037/tlcr-20-1191

\section{Introduction}

Malignant pleural effusion (MPE) is a common presentation in clinical practice caused by advanced malignant diseases. Cancer patients with MPE usually manifest breathlessness with a poor survival rate of 3-12 months (1). The standard management for symptomatic MPE is pleural space intervention to relieve discomfort and reduce 
pleural fluid recurrence (2). Various metastatic tumors can lead to MPE, among which lung cancer accounts for the largest proportion, followed by breast cancer and hematological neoplasms (3). Porcel et al. reported that MPE is an independent risk factor for non-small cell lung cancer (NSCLC) patients with a poor prognosis (3). The overall survival (OS) of NSCLC patients with MPE is approximately 5.5 months, which is shorter than mesothelioma patients with MPE $(4,5)$. Identifying risk factors that affect prognosis can help lung cancer patients with MPE receive optimal individualized treatment. Clive et al. developed a prognostic model named the LENT score [pleural fluid lactate dehydrogenase, Eastern Cooperative Oncology Group (ECOG) performance score (PS), neutrophil-to-lymphocyte ratio, and tumor type] to predict the survival of MPE, in which high lactic dehydrogenase (LDH) in pleural fluid, ECOG PS score, high neutrophilto-lymphocyte ratio (NLR) and lung cancer indicated poor survival (6). A multicohort analysis combined biological and clinical parameters to finally build a PROMISE (prognostic and therapeutic markers of malignant pleural effusion) model to estimate patients' mortality with MPE (7).

However, the LENT and PROMISE scores are models that predict the prognosis of all malignant tumors, which may reduce the accuracy of specific types of malignancy. Lung cancer is the leading cause of cancer-related death, with an estimated annual mortality of 631 thousand in China alone in 2015 (8). Few studies have identified prognostic factors of patients with MPE. A retrospective study by Kasapoglu et al. found that high serum C-reactive protein (CRP), low serum albumin, lower pleural protein, and distant metastasis predicted shorter survival in NSCLC patients with MPE (9). Pleural LDH and NLR were demonstrated to be clinical biomarkers for prognostication in lung cancer patients $(10,11)$. Biological parameters like pleural MAC30 (over-expressed meningioma-associate protein) and VEGF (vascular endothelial growth factor) in pleural effusion were predictive for the survival of lung cancer patients with MPE $(12,13)$. However, with oncogenic driver mutations and immunotherapy, these predictive biomarkers and models may underestimate the outcome of these populations and deepen the prediction inaccuracy $(14,15)$. Therefore, it is imperative to develop a predictive model to estimate the survival of lung cancer patients with MPE.

This study was designed to generate a predictive score in selected lung cancer patients with MPE derived from the combination of independent risk factors of death.
We present the following article in accordance with the TRIPOD (transparent reporting of a multivariable prediction model for individual prognosis or diagnosis) reporting checklist (available at http://dx.doi.org/10.21037/ tlcr-20-1191).

\section{Methods}

\section{Ethics statement}

The study was conducted in accordance with the Declaration of Helsinki (as revised in 2013) and approved by the local ethics committee of Jinling Hospital (registration ID. DBNJ329). Informed consent from individuals was waived based on the retrospective nature of this study.

\section{Participants}

Lung cancer patients with MPE in Nanjing Jinling Hospital from January 2008 to June 2018 were recruited according to the following criteria: (I) aged $18-80$ years old; (II) pathological diagnosis of lung cancer; (III) histological or cytological confirmation of malignant pleural effusion; (IV) had received active therapy after the first episode of MPE. These eligible patients were followed up, and survival time was calculated from the first episode of MPE to death. Data regarding age, gender, smoking history, ECOG PS, relapse or not, white blood cell (WBC), NLR, platelet, CRP, serum LDH, serum albumin and globulin, pleural LDH, pathology, tumor, node, metastasis (TNM) stage, and oncogenic driver mutations were collected. Patients with incomplete data were excluded from this study.

\section{Statistical analysis}

Data were analyzed using the statistical software SPSS version 22 (IBM Corp., Chicago, IL, USA). Risk factors affecting prognosis were extracted using the chi-square test and univariate Kaplan-Meier analysis. Variables with a 2 -sided $\mathrm{P}$ value of less than 0.05 were selected for multivariate Cox proportional-hazards analysis. Different points were given to risk factors according to the RR (risk ratio) value. According to the sum of all risk factors, lung cancer patients were categorized into 3 groups, which were low-risk, moderate-risk, and high-risk. The prognostic score's sensitivity and specificity were tested by the receiver operating characteristic (ROC) curve. 
Table 1 Demographic characteristics of lung cancer patients with MPE

\begin{tabular}{lc}
\hline Characteristics & N (Total $=367)(\%)$ \\
\hline Mean age $(\mathrm{y})$ & $59.1(23-85)$ \\
$\geq 65$ & $122(33.2)$ \\
$<65$ & $245(66.8)$ \\
Gender & \\
Male & $219(59.7)$ \\
Female & $148(40.3)$ \\
Smokers & \\
Yes & $171(46.6)$ \\
No & $196(53.4)$ \\
Histological type & \\
Adenocarcinoma & $284(77.4)$ \\
Squamous carcinoma & $34(9.2)$ \\
Small/large cell lung cancer & $41(11.2)$ \\
Adenosquamous carcinoma & $8(2.2)$ \\
ECOG PS & \\
0-1 & $307(83.7)$ \\
$\geq 2$ & $60(16.3)$ \\
Nelapse & \\
\hline
\end{tabular}

ECOG PS, Eastern Cooperative Oncology Group performance score.

\section{Results}

From January 2008 to June 2018, 367 lung cancer patients diagnosed with MPE in Nanjing Jinling Hospital were recruited to the development cohort. The demographic characteristics are shown in Table 1. Fifty-four participants were lost to follow up, and 22 participants stayed alive during the follow-up period. Of the 367 participants, the mean age was 59.1 years, $59.7 \%$ were males, and $53.4 \%$ were non-smokers. Adenocarcinoma accounted for most lung cancers $(77.4 \%)$, followed by small or large cell carcinoma and squamous carcinoma. Poor performance status with ECOG PS greater than or equal to 2 was observed in $16.3 \%$ of the participants. Of the population, $61.6 \%$ were first diagnosed with lung cancer in the first episode of MPE, while $38.4 \%$ represented relapse or lung

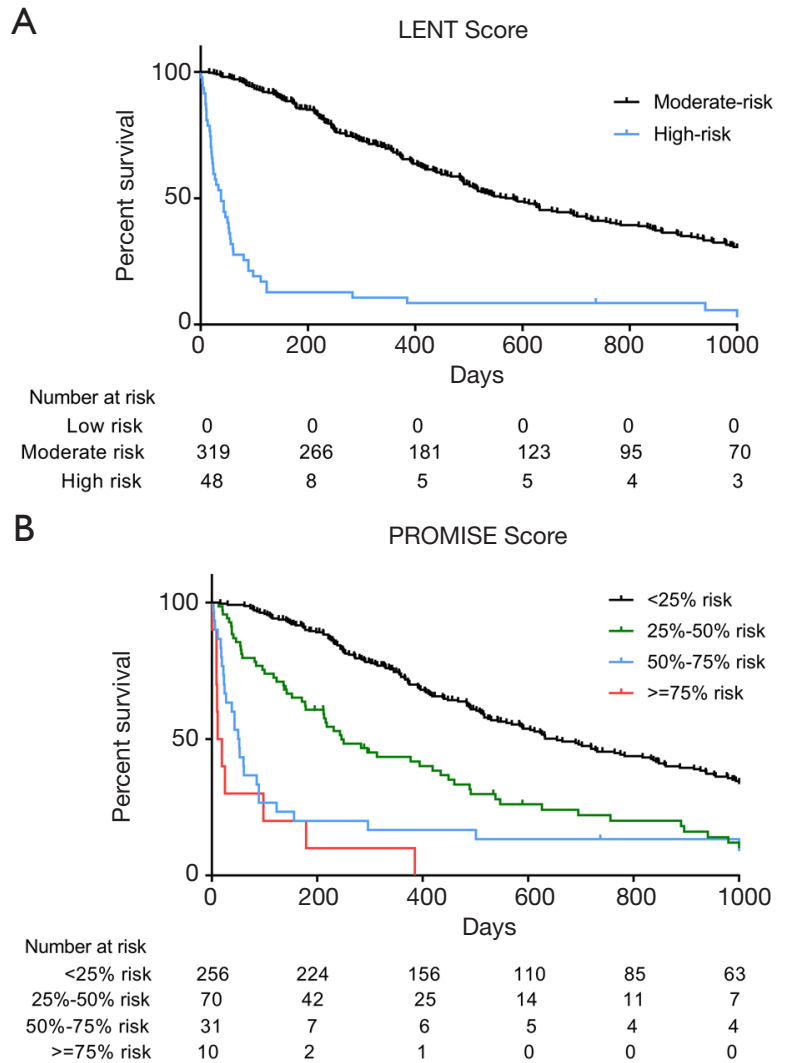

Figure 1 Performance of LENT score and PROMISE score in lung cancer patients with MPE. (A) Kaplan-Meier survival curves from lung cancer patients with low, moderate, and high LENT score. (B) Kaplan-Meier survival curves from lung cancer patients with four risk groups of PROMISE score .

cancer progression since MPE had first occurred.

Kaplan-Meier survival analysis was conducted using 367 lung cancer participants. The LENT score was used to categorize participants into moderate-risk and high-risk groups, as lung cancer was designated a high-risk tumor type with at least 2 points (Figure 1A). The PROMISE model's survival curves and the $50-75 \%$ risk group and $>75 \%$ risk group overlapped, as shown in Figure $1 B$.

We used univariate analysis to select variables to enter the multivariate Cox proportional-hazards model with the conditional forward method. A total of 12 risk factors were confirmed to be significant by univariate analysis, of which 5 were selected as the predictors of survival (Table 2). Relapse or not, ECOG PS, CRP, pleural LDH, and stage maintained the prognostic significance, and the RR values are also listed in Table 2. Each predictive factor was assigned corresponding scores according to their RR values. Then, lung cancer 
Table 2 Multivariate cox analysis of the development cohort of lung cancer patients with MPE and scoring system

\begin{tabular}{lccc}
\hline Risk factor & P value & RR & Points \\
\hline Relapse & 0.001 & 1.530 & 3 \\
ECOG PS & $<0.001$ & 2.912 & 5 \\
CRP & $<0.001$ & 1.006 & 2 \\
LDH & 0.011 & 1.000 & 2 \\
Stage & $<0.001$ & 1.957 & 3 \\
\hline
\end{tabular}

Table 3 The RECLS score calculation for lung cancer patients with MPE

\begin{tabular}{|c|c|}
\hline Variable & Score \\
\hline \multicolumn{2}{|l|}{ Relapse } \\
\hline No & 0 \\
\hline Yes & 3 \\
\hline \multicolumn{2}{|l|}{ ECOG PS } \\
\hline $0-1$ & 0 \\
\hline$\geq 2$ & 5 \\
\hline \multicolumn{2}{|l|}{ CRP (mg/L) } \\
\hline$<100$ & 0 \\
\hline$\geq 100$ & 2 \\
\hline \multicolumn{2}{|c|}{ LDH in pleural fluid (U/L) } \\
\hline$<1,500$ & 0 \\
\hline$\geq 1,500$ & 2 \\
\hline \multicolumn{2}{|l|}{ Stage } \\
\hline IVA & 0 \\
\hline IVB & 3 \\
\hline \multicolumn{2}{|l|}{ Risk categories } \\
\hline Low risk & $0-4$ \\
\hline Moderate risk & $5-10$ \\
\hline High risk & $11-15$ \\
\hline
\end{tabular}

ECOG PS, Eastern Cooperative Oncology Group performance score; CRP, C-reaction protein; $\mathrm{LDH}$, lactate dehydrogenase;

patients were divided into 3 groups after adding up the points: low-risk ( $0-4$ points), moderate-risk ( $5-10$ points), and high-risk (11-15 points) (Table 3 and Figure 2).

Of the 367 lung cancer participants, 249 were categorized

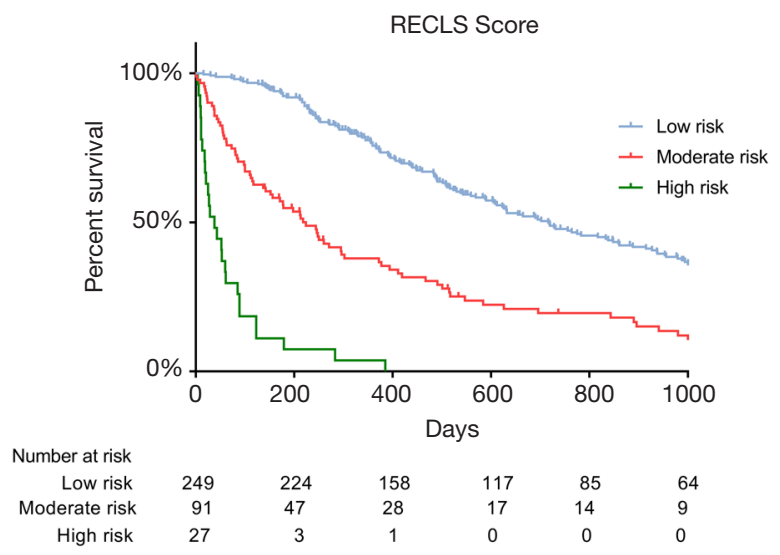

Figure 2 Kaplan-Meier survival curves for five variables (RECLS score) in lung cancer patients with MPE in development cohort.

as the low-risk group with a median survival of 716 days. Survival rates of this population at 1,6 , and 12 months were $99.2 \%, 92.3 \%$, and $75.7 \%$, respectively. Participants in the high-risk group had a significantly shorter median survival (38 days), and $51.9 \%, 7.4 \%$, and $3.7 \%$ of these participants survived to 1,6 , and 12 months, respectively. The moderate-risk group showed intermediate prognoses of $90.1 \%, 54.8 \%$, and $37.9 \%$ at 1,6 , and 12 months, with a median survival of 224 days (Table S1).

The ROC analysis of the RECLS score in lung cancer participants is shown in Figure 3. The AUC values for the RECLS model were $0.911,0.845$, and 0.754 , respectively, at 1,6 , and 12 months. The high AUC values demonstrated the high sensitivity of the RECLS score.

Similarly, we selected 7 variables using the same statistic methods to estimate lung adenocarcinoma participants' survival with MPE. We added oncogenic driver mutations to multivariate Cox proportional-hazards analysis. Except for the 5 risk factors above (relapse or not, ECOG PS, CRP, pleural LDH, and TNM stage), ALB (albumin/ globulin ratio), and oncogenic driver mutations were also confirmed be significant to the mortality rate. We made a RECLSAM score proportional to each variable's $\mathrm{RR}$ values (Tables 4,5). The survival of the low-risk, moderate-risk, and high-risk groups is shown in Figure 4. The median survival of the low-risk, moderate-risk, and high-risk populations were 783 days, 227 days, and 38 days, respectively. The survival proportions of 1,6 , and 12 months are listed in Table S2. The ROC curves of RECLSAM are also displayed in Figure S1. 

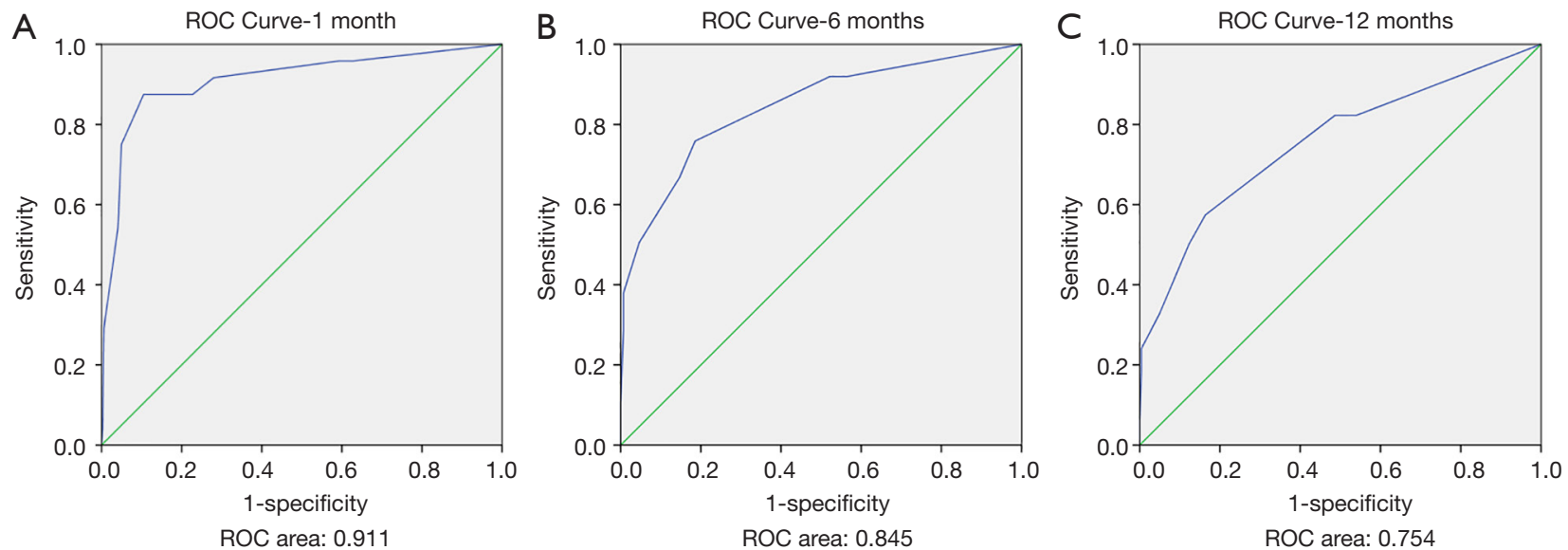

Figure 3 Receiver operating characteristic (ROC) curve analysis for the RECLS score. (A) Development cohort at 1 month. (B) Development cohort at 6 months. (C) Development cohort at 12 months.

Table 4 Multivariate cox analysis of the development cohort of lung adenocarcinoma patients with MPE and Scoring System

\begin{tabular}{lccc}
\hline Risk factor & P value & RR & Points \\
\hline Relapse & 0.001 & 1.53 & 3 \\
ECOG PS & $<0.001$ & 2.912 & 5 \\
CRP & $<0.001$ & 1.006 & 2 \\
A/G & 0.007 & 0.609 & 1 \\
LDH & 0.011 & 1 & 2 \\
Stage & $<0.001$ & 1.957 & 3 \\
Mutation & 0.013 & 0.705 & 1 \\
\hline
\end{tabular}

ECOG PS, Eastern Cooperative Oncology Group performance score; CRP, C-reaction protein; LDH, lactate dehydrogenase; A/G, Albumin/Globulin ratio.

\section{Discussion}

It is common for MPE to present in lung cancer patients. Survival prediction of lung cancer patients with MPE can assist in the selection of individualized treatment. Thus, developing a new predictive model to predict the outcome of lung cancer patients with MPE is needed. We discovered the prognostic scores (RECLS score and RECLSAM score) for the selected type of lung cancer patients with MPE and lung adenocarcinoma patients with MPE. The 5 and 7 clinical variables were confirmed as independent risk factors for the survival of pulmonary carcinoma participants with MPE, which could help physicians and families make subsequent individual treatment decisions.
Also, the LENT score was performed in the development cohort with only 2 curves, which might not distinguish the low-risk population from lung cancer patients. The PROMISE model also had limitations in discriminating the 4 groups completely. Both the LENT and PROMISE prognostic models applied to unselected cancer patients, which might decline the accuracy of predicting a particular tumor type. Hence, our study included the development of new prognostic models, including 5 and 7 variables.

The first appearance of MPE usually indicated a new diagnosis or recurrence of lung cancer. We found relapse was a strong predictive variable for poor survival as it signaled that the disease had continued to progress after receiving prior treatment. These relapse patients needed a second line treatment to control the progression. This was consistent with chemotherapy and radiotherapy's risk factors as reported by the PROMISE model (7). Multivariable analysis confirmed that high ECOG PS was an independent risk factor of survival. Previous studies have also reported that PS was associated with MPE prognosis derived from several types of carcinoma (16-18). Poor PS indicated limited therapeutic effects and short OS. The common biomarker of the systemic inflammatory response, CRP, was a prognostic factor in our study. Kasapoglu et al. also confirmed that high serum CRP was associated with poor survival among NSCLC patients (9). Inflammation was reported to be a hallmark feature of tumor development, which played a role in cancer immune response $(19,20)$. Heikkilä outlined possible mechanisms of inflammation and cancer: tissue inflammation occurred as the tumor grew, and serum CRP increased; CRP was a product of the immune 
Table 5 The RECLSAM score calculation for lung adenocarcinoma patients with MPE

\begin{tabular}{|c|c|}
\hline Variable & Score \\
\hline \multicolumn{2}{|l|}{ Relapse } \\
\hline No & 0 \\
\hline Yes & 3 \\
\hline \multicolumn{2}{|l|}{ ECOG PS } \\
\hline $0-1$ & 0 \\
\hline$\geq 2$ & 5 \\
\hline \multicolumn{2}{|l|}{ CRP (mg/L) } \\
\hline$<100$ & 0 \\
\hline$\geq 100$ & 2 \\
\hline \multicolumn{2}{|c|}{ LDH in pleural fluid (U/L) } \\
\hline$<1,500$ & 0 \\
\hline$\geq 1,500$ & 2 \\
\hline \multicolumn{2}{|l|}{ Stage } \\
\hline IVA & 0 \\
\hline IVB & 3 \\
\hline \multicolumn{2}{|c|}{ A/G (albumin/globulin) } \\
\hline$\geq 1.5$ & 0 \\
\hline$<1.5$ & 1 \\
\hline \multicolumn{2}{|l|}{ Mutation } \\
\hline Yes & 0 \\
\hline No & 1 \\
\hline \multicolumn{2}{|l|}{ Risk categories } \\
\hline Low risk & $0-4$ \\
\hline Moderate risk & $5-11$ \\
\hline High risk & $12-17$ \\
\hline
\end{tabular}

ECOG PS, Eastern Cooperative Oncology Group performance score; CRP, C-reaction protein; LDH, lactate dehydrogenase; $\mathrm{A} / \mathrm{G}$, albumin/globulin ratio.

response toward tumor antigens (21). As an inflammatory marker, NLR has also been considered related to lung cancer patients with MPE (22). However, there was no statistically significant difference between the high NLR and low NLR groups in our research using multivariable Cox analysis. Like the LENT score, the RECLS score also found that LDH in pleural fluid was responsible for lung cancer patients' survival. A retrospective study, including

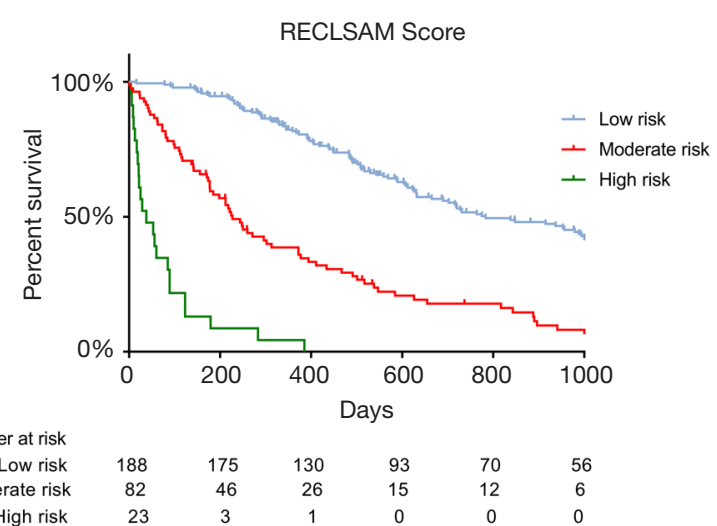

Figure 4 Kaplan-Meier survival curves for seven variables (RECLSAM Score) in lung adenocarcinoma patients with MPE in development cohort.

74 lung adenocarcinoma patients with MPE, reported that subjects with higher pleural LDH $(>1,500 \mathrm{IU} / \mathrm{L})$ had shorter survival than relatively lower pleural LDH $(\leq 1,500$ IU/L) (10). Elevated pleural LDH may be associated with glycolysis over oxidative phosphorylation for energy by cancer cells (23). The TNM stage was also shown to have the ability to predict the prognosis of lung cancer participants in the present study. According to the $8^{\text {th }}$ edition, the main point of discrimination between stage IVA and stage IVB was multiple distant metastases. Participants were classified into stage IVB when there was a minimum of 2 distant metastatic foci. Previous research has also indicated that distant metastasis was an independent risk factor for the survival of NSCLC patients with MPE (9); however, the number of metastatic foci was not explained in detail. Unexpectedly, the pathological pattern was not an independent variable related to the survival rate. To our knowledge, small cell lung cancer (SCLC) is aggressive cancer with shorter survival than NSCLC (24). The insignificant difference may have been due to the small proportion of SCLC among lung cancer participants.

We then developed a predictive model for the subgroup of lung adenocarcinoma participants. In addition to the 5 variables of the RECLS model, albumin to globulin ratio $(\mathrm{A} / \mathrm{G})$ and mutation were also clinical biomarkers. Serum $\mathrm{A} / \mathrm{G}$ was previously reported to affect the prognosis of NSCLC patients $(25,26)$. Targeted therapy has led to a new era for lung adenocarcinoma, and gene mutation detection is recommended in the National Comprehensive Cancer Network (NCCN) guidelines (27). Abisheganaden et al. have confirmed that the epidermal growth factor 
receptor (EGFR) mutation was a protective factor for lung adenocarcinoma patients (28).

We used ROC curves to assess the sensitivity and specificity of the RECLS and RECLSAM scores. The 2 prognostic models were valuable and efficient in predicting lung cancer and lung adenocarcinoma patients' survival. It was essential to enable discrimination of the different risks of lung cancer patients with MPE to facilitate optimal management strategy selection by doctors and. Those belonging to high-risk groups might receive conservative, symptomatic treatment, while low-risk patients can opt for more aggressive management.

The strengths of this research include the large sample size and clinical practicality of noninvasive risk factor assessment. We developed predictive models of survival in the selected populations of lung cancer and lung adenocarcinoma with MPE.

Our research had several limitations. Firstly, this was a retrospective double-centered study spanning from 2008 to 2018. Only a few patients were still alive by the followup time. Secondly, we could not control the treatment options since therapy usually changed according to the severity of diseases and their adverse effects. Also, we used dichotomized variables, which may have decreased the accuracy of continuous variables.

\section{Conclusions}

We developed prognostic models to predict the survival of lung cancer patients with MPE. The RECLS score was developed for lung cancer patients with MPE, and the RECLSAM score especially for lung adenocarcinoma patients with MPE. The 2 models were shown to be easy to calculate and practical to use in clinical work, which may be valuable for clinicians in selecting optimal management strategies.

\section{Acknowledgments}

Funding: This work was supported by grants from the National Natural Science Foundation of China (grant number 81401903,81572937 and 81572273 ); the $16^{\text {th }}$ batch "Summit of the Six Top Talents" Program of Jiangsu Province (grant number WSN-154); China Postdoctoral Science Foundation $12^{\text {th }}$ batch Special fund (Postdoctoral number: 45786); China Postdoctoral Science Foundation $64^{\text {th }}$ batch (Postdoctoral number: 45786); Jiangsu Provincial Postdoctoral Science Foundation (grant number
2018K049A); the Natural Science Foundation of Jiangsu province (grant number BK20180139 and BK20161386); Jiangsu Provincial Medical Youth Talent (grant number QNRC2016125), and the Nanjing Medical Science and Technology Development Project (No. ZKX17044), the Jiangsu Provincial Key Research and Development Program (No. BE2016721).

\section{Footnote}

Reporting Checklist: The authors have completed the TRIPOD reporting checklist. Available at http://dx.doi. org/10.21037/tlcr-20-1191

Data Sharing Statement: Available at http://dx.doi. org/10.21037/tlcr-20-1191

Peer Review File: Available at http://dx.doi.org/10.21037/ tlcr-20-1191

Conflicts of Interest: All authors have completed the ICMJE uniform disclosure form (available at http://dx.doi. org/10.21037/tlcr-20-1191). YS serves as an editor-in-chief of Translational Lung Cancer Research from Sep 2020 to Aug 2022. TL serves as an unpaid associate editor-in-chief of Translational Lung Cancer Research. The other authors have no conflicts of interest to declare.

Ethical Statement: The authors are accountable for all aspects of the work in ensuring that questions related to the accuracy or integrity of any part of the work are appropriately investigated and resolved. The study was conducted in accordance with the Declaration of Helsinki (as revised in 2013) and approved by the local ethics committee of Jinling Hospital (registration ID. DBNJ329). Informed consent from individuals was waived based on the retrospective nature of this study.

Open Access Statement: This is an Open Access article distributed in accordance with the Creative Commons Attribution-NonCommercial-NoDerivs 4.0 International License (CC BY-NC-ND 4.0), which permits the noncommercial replication and distribution of the article with the strict proviso that no changes or edits are made and the original work is properly cited (including links to both the formal publication through the relevant DOI and the license). See: https://creativecommons.org/licenses/by-nc-nd/4.0/. 


\section{References}

1. Roberts ME, Neville E, Berrisford RG, et al. Management of a malignant pleural effusion: British Thoracic Society pleural disease guideline 2010. Thorax 2010;65:Suppl 2:ii32-ii40.

2. Bibby AC, Dorn P, Psallidas I, et al. ERS/EACTS statement on the management of malignant pleural effusions. Eur Respir J 2018;27:52:1800349.

3. Porcel JM, Esquerda A, Vives M, et al. Etiology of pleural effusions: analysis of more than 3,000 consecutive thoracenteses. Arch. Bronconeumol 2014;50:161-5.

4. William WN, Lin HY, Lee JJ, et al. Revisiting stage IIIB and IV non-small cell lung cancer: analysis of the surveillance, epidemiology, and end results data. Chest 2009;136:701-9.

5. Scherpereel A, Astoul P, Baas P, et al. Guidelines of the European Respiratory Society and the European Society of Thoracic Surgeons for the management of malignant pleural mesothelioma. Eur Respir J 2010;35:479-495.

6. Clive AO, Kahan BC, Hooper CE, et al. Predicting survival in malignant pleural effusion: development and validation of the LENT prognostic score. Thorax 2014;69:1098-104.

7. Psallidas I, Kanellakis NI, Gerry S, et al. Development and validation of response markers to predict survival and pleurodesis success in patients with malignant pleural effusion (PROMISE): a multicohort analysis. Lancet Oncol 2018;19:930-9.

8. Zheng RS, Sun KX, Zhang SW, et al. Report of cancer epidemiology in China, 2015. Zhonghua Zhong Liu Za Zhi 2019;41:19-28.

9. Kasapoglu US, Arınç S, Gungor S, et al. Prognostic factors affecting survival in non-small cell lung carcinoma patients with malignant pleural effusions. Clin Respir J 2016;10:791-9.

10. Verma A, Phua CK, Sim WY, et al. Pleural LDH as a prognostic marker in adenocarcinoma lung with malignant pleural effusion. Medicine (Baltimore) 2016;95:e3996.

11. Yao Y, Yuan D, Liu H, et al. Pretreatment neutrophil to lymphocyte ratio is associated with response to therapy and prognosis of advanced non-small cell lung cancer patients treated with first line platinum-based chemotherapy. Cancer Immunol Immunother 2013;62:471-9.

12. Shan Y, Ding H, Lu J, et al. Pleural MAC30 as a prognostic marker in NSCLC with malignant pleural effusion. Oncotarget 2017;8:112809-15.

13. Zhang Y, Yu LK, Lu GJ, et al. Prognostic values of VEGF and endostatin with malignant pleural effusions in patients with lung cancer. Asian Pac J Cancer Prev 2014;15:8435-40.

14. Wu SG, Yu CJ, Tsai MF, et al. Survival of lung adenocarcinoma patients with malignant pleural effusion. Eur Respir J 2013;41:1409-18.

15. Gubens MA. NCCN Guidelines Updates: New Immunotherapy Strategies for Improving Outcomes in Non-Small Cell Lung Cancer. Presented at: 2019 NCCN Annual Conference; March 21-23, 2019.

16. Bielsa S, Salud A, Martinez M, et al. Prognostic significance of pleural fluid data in patients with malignant effusion. Eur J Intern Med 2008;19:334-9.

17. Anevlavis S, Kouliatsis G, Sotiriou I, et al. Prognostic factors in patients presenting with pleural effusion revealing malignancy. Respiration 2014;87:311-6.

18. Sánchez-Muñoz A 1, Pérez-Ruiz E, Sáez MI, et al. Limited impact of palliative chemotherapy on survival in advanced solid tumors in patients with poor performance status. Clin Transl Oncol 2011;13:426-9.

19. Hanahan D, Weinberg RA. Hallmarks of cancer: the next generation. Cell 2011;144:646-74.

20. Diakos CI, Charles KA, McMillan DC, et al. Cancerrelated inflammation and treatment effectiveness. Lancet Oncol 2014;15:e493-503.

21. Heikkilä K, Ebrahim S, Lawlor DA. A systematic review of the association between circulating concentrations of $\mathrm{C}$ reactive protein and cancer. J Epidemiol Community Health 2007;61:824-33.

22. Lee YS, Nam HS, Lim JH, et al. Prognostic impact of a new score using neutrophil-to-lymphocyte ratios in the serum and malignant pleural effusion in lung cancer patients. BMC Cancer 2017;17:557.

23. Verma A, Abisheganaden J, Light RW, et al. Identifying malignant pleural effusion by a cancer ratio (serum LDH: pleural flfluid ADA ratio). Lung 2016;194:147-53.

24. Powell HA, Tata LJ, Baldwin DR, et al.Treatment decisions and survival for people with small-cell lung cancer.Br J Cancer 2014;110:908-15.

25. Yao Y, Zhao M, Yuan D, et al. Elevated pretreatment serum globulin albumin ratio predicts poor prognosis for advanced non-small cell lung cancer patients. J Thorac Dis 2014;6:1261-70.

26. Duran AO, Inanc M, Karaca H, et al. Albumin-globulin ratio for prediction of long-term mortality in lung adenocarcinoma patients. Asian Pac J Cancer Prev 2014;15:6449-53.

27. Ettinger DS, Aisner DL, Wood DE, et al. NCCN Guidelines Insights: Non-Small Cell Lung Cancer, Version 
5.2018. J Natl Compr Canc Netw 2018;16:807-21.

28. Abisheganaden J, Verma A, Dagaonkar RS, et al. An Observational Study Evaluating the Performance of
LENT Score in the Selected Population of Malignant

Pleural Effusion from Lung Adenocarcinoma in Singapore. Respiration 2018;96:308-13.

Cite this article as: Zhang T, Chen X, Wan B, Xu Y, Liu H, Lv T, Zhan P, Song Y. Development of RECLS score to predict survival in lung cancer patients with malignant pleural effusion. Transl Lung Cancer Res 2021;10(3):1318-1326. doi: 10.21037/ tlcr-20-1191 


\section{Supplementary}
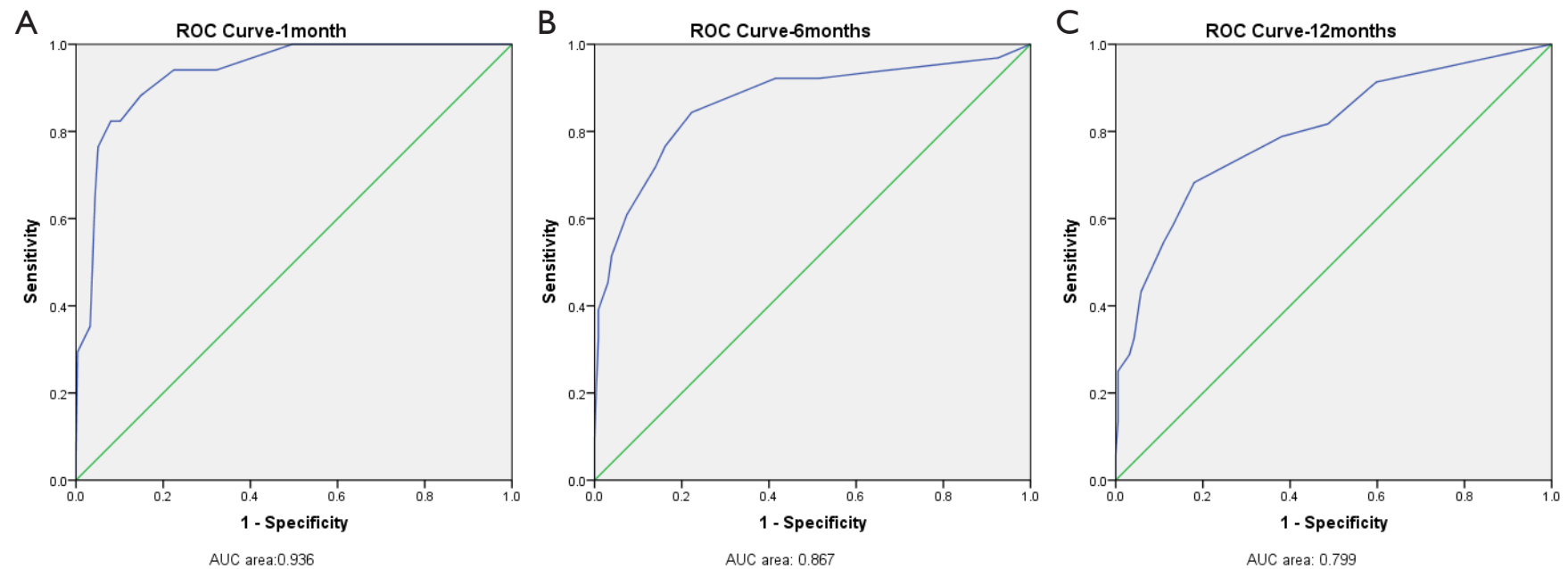

Figure S1 Receiver operating characteristic (ROC) curve analysis for the RECLSAM score. (A) Development cohort at 1 month. (B) Development cohort at 6 months. (C) Development cohort at 12 months.

Table S1 Median survival and survival rate in lung cancer patients with MPE, according to risk category of RECLS score

\begin{tabular}{lccccc}
\hline Groups & Median survival (d) & Survival at $1 \mathrm{~m}$ & Survival at 3 $\mathrm{m}$ & Survival at 6 m & Survival at 1 yr \\
\hline Low risk & 716 & $99.20 \%$ & $97.99 \%$ & $92.32 \%$ & $75.70 \%$ \\
Moderate risk & 224 & $90.11 \%$ & $70.33 \%$ & $54.76 \%$ & $37.91 \%$ \\
High risk & 38 & $51.85 \%$ & $18.52 \%$ & $7.41 \%$ & $3.70 \%$ \\
\hline
\end{tabular}

Table S2 Median survival and survival rate in lung adenocarcinoma patients with MPE, according to risk category of RECLSAM score

\begin{tabular}{lccccc}
\hline Groups & Median survival $(\mathrm{d})$ & Survival at 1 $\mathrm{m}$ & Survival at 3 $\mathrm{m}$ & Survival at 6 m & Survival at 1 yr \\
\hline Low risk & 783 & $99.47 \%$ & $98.94 \%$ & $94.65 \%$ & $81.71 \%$ \\
Moderate risk & 227 & $93.90 \%$ & $78.05 \%$ & $59.46 \%$ & $38.63 \%$ \\
High risk & 38 & $52.17 \%$ & $21.74 \%$ & $8.70 \%$ & $4.35 \%$ \\
\hline
\end{tabular}

